\title{
Haar null and non-dominating sets
}

\author{
by \\ Sławomir Solecki (Bloomington, IN)
}

\begin{abstract}
We study the $\sigma$-ideal of Haar null sets on Polish groups. It is shown that on a non-locally compact Polish group with an invariant metric this $\sigma$-ideal is closely related, in a precise sense, to the $\sigma$-ideal of non-dominating subsets of $\omega^{\omega}$. Among other consequences, this result implies that the family of closed Haar null sets on a Polish group with an invariant metric is Borel in the Effros Borel structure if, and only if, the group is locally compact. This answers a question of Kechris. We also obtain results connecting Haar null sets on countable products of locally compact Polish groups with amenability of the factor groups.
\end{abstract}

1. Introduction. A subset $A$ of a Polish group $G$ is called Haar null if it is contained in a universally measurable set $B$ for which there exists a Borel probability measure $\mu$ on $G$ such that $\mu(g B h)=0$ for all $g, h \in G$. This family of sets is closed under translations (simultaneously from left and right), taking subsets, and countable unions. The notion of Haar null sets is a natural extension of the notion of sets of Haar measure zero: if $G$ happens to be locally compact, then Haar null sets are precisely the sets of Haar measure zero. Since the publication of Christensen's paper [C1] which introduced this new notion, Haar null sets have found many applications. For example, they were used to find a generalization to Banach spaces of Rademacher's differentiability theorem [C2], and implicitly [Ma, Theorem 4.5]. Most recently some very interesting connections have been found between reflexivity of separable Banach spaces and Haar nullness of closed, convex, nowhere dense sets [MS, M1, M2, M3] with the final result in [M3]. Also a lot of effort has been put into determining whether or not certain concrete sets are Haar null (see for example [D, DM, Hu, HSY, ST]).

The class of groups we will consider in the first part of the paper is the class of Polish groups with an invariant metric, that is, Polish groups $G$

2000 Mathematics Subject Classification: 28C10, 03E15, 43A07.

Research supported by NSF grant DMS-9803676. 
which admit a metric $d$ such that $d(g x h, g y h)=d(x, y)$ for any $g, h, x, y \in G$. All Polish abelian groups admit invariant metrics and there exist nonabelian groups with invariant metrics. The results in this paper are new also for abelian groups and, in fact, even for separable Banach spaces.

The present paper is concerned with a circle of problems and results, initiated in [C1], which have to do with comparing properties of the $\sigma$-ideal of Haar null sets on non-locally compact groups with the properties of Haar null sets on locally compact groups, that is, with properties of Haar measure zero sets. There were indications that the locally compact case and the non-locally compact case are rather different. For example, if $G$ is not locally compact and has an invariant metric, then each compact subset of $G$ is Haar null (see [C1] for abelian groups and [D] in general) and the $\sigma$-ideal of Haar null sets does not have the countable chain condition (that is, there exists an uncountable family of pairwise disjoint Borel sets each of which is not Haar null (cf. [D] for a large subclass of Polish abelian groups, $[\mathrm{S}]$ in general). Classical theorems state that negations of these statements hold for Polish locally compact groups. There was one aspect of this comparison, first pointed out by Kechris, that remained unresolved: he asked whether the family of closed Haar null subsets of a Polish group is Borel in the Effros Borel structure. It is so in the case when $G$ is locally compact and it was not known to be non-Borel on any non-locally compact group.

We prove a theorem which tries to explain these dissimilarities and answers Kechris' question. We show that the $\sigma$-ideal of non-dominating subsets of $\omega^{\omega}$ can be "reduced" to the $\sigma$-ideal of Haar null sets on a non-locally compact Polish group with an invariant metric. This is rather unexpected since the $\sigma$-ideal of non-dominating sets is very much unlike the $\sigma$-ideal of measure zero sets with respect to a Borel $\sigma$-finite measure. Actually, if this new notion of reduction is naturally generalized to compare arbitrary ideals on Polish spaces, then one easily shows that the $\sigma$-ideal of non-dominating sets is never reducible to the $\sigma$-ideal of measure zero sets with respect to any $\sigma$-finite Borel measure. The notion of "reduction" used here is reminiscent of the notion of the Rudin-Keisler or even Rudin-Blass reduction used to compare ideals of subsets of $\omega$ and can be considered to be its continuous analogue. As a consequence of our reduction result we deduce that the family of closed Haar null sets can be non-Borel. In fact, we show that Borelness of the family of closed Haar null sets characterizes local compactness among Polish groups with invariant metric. Furthermore, it is straightforward to deduce from this result Haar nullness of compact sets and the failure of the countable chain condition mentioned above and proved earlier in [C1], [D], and $[\mathrm{S}]$. We also deduce some estimates on the additivity and cofinality of the Haar null $\sigma$-ideal. 
It is also interesting, when comparing properties of Haar null sets on locally compact and non-locally compact groups, to consider groups which, though not locally compact, are obtained from locally compact ones by a simple operation; for example, countable products of locally compact groups. We study to what extent the Haar null $\sigma$-ideal on those product groups is determined by Haar measures on the factor groups. Surprisingly, the notion of amenability seems to be relevant here. It turns out that a subset of a countable product of locally compact amenable groups is Haar null precisely when its Haar nullness is witnessed by a measure which is the product of probability measures on the factor groups with each of these measures equivalent to the appropriate Haar measure.

2. Haar null sets and non-dominating sets. We think of $\omega^{\omega}$, the space of all functions from the set of all natural numbers to itself, as equipped with a partial order defined as follows. For $x, y \in \omega^{\omega}$, put $x \leq^{*} y$ if $x(n) \leq y(n)$ for all but finitely many $n \in \omega$. Recall that a subset $A$ of $\omega^{\omega}$ is dominating if for any $x \in \omega^{\omega}$ there exists a $y \in A$ such that $x \leq^{*} y$. So, a subset is non-dominating if for some $x$ for each $y \in A, y(n)<x(n)$ holds for infinitely many $n$ 's. Note that non-dominating sets constitute a $\sigma$-ideal.

Given two Polish spaces $X$ and $Y$ with $\sigma$-ideals $I$ and $J$ on $X$ and $Y$, respectively, we would consider $J$ to be simpler than $I$ if it can be reduced to $I$ in the following natural way: for some Borel set $B \subseteq X$ and some Borel function $f: B \rightarrow Y$, we have $A \in J \Leftrightarrow f^{-1}(A) \in I$. This notion of reduction can be thought of as a generalization of the Rudin-Keisler reduction which is used to compare ideals, or dually filters, of subsets of $\omega$. This new notion has some nice properties; for example, it is transitive. It is also meaningful, that is, there exist $\sigma$-ideals $I$ and $J$ such that $J$ is not reducible to $I$. A particularly important, from the point of view of the problems of this paper, example of this is obtained by taking $I$ to be the $\sigma$-ideal of measure zero sets with respect to some $\sigma$-finite Borel measure defined on a Polish space $X$ and letting $J$ be the $\sigma$-ideal of non-dominating subsets of $\omega^{\omega}$. That $J$ cannot be reduced to $I$ can be seen by remarking that there exist $A_{\alpha} \subseteq \omega^{\omega}$, $\alpha \in 2^{\omega}$, Borel pairwise disjoint sets none of which is in $J$, and then noticing that if $f$ were a reduction, then $f^{-1}\left(A_{\alpha}\right)$ would be a family of Borel pairwise disjoint subsets of $X$ of positive measure, and such families do not exist. In contrast to this fact, we show below that the $\sigma$-ideal of non-dominating sets is reducible to the $\sigma$-ideal of Haar null sets on a non-locally compact Polish group with an invariant metric. In fact, we will be able to do much better: the function $f$ can be taken to be a continuous open surjection and its domain can be taken to be closed and large in a suitable sense. (Actually, one can even get $f$ to be such that preimages of compact sets are compact. 
So this reduction resembles more the stronger than Rudin-Keisler notion of reduction between ideals of subsets of $\omega$ called the Rudin-Blass reduction; see $[\mathrm{LZ}]$.

Theorem 2.1. Let $G$ be a Polish non-locally compact group with an invariant metric. There exist a closed set $F \subseteq G$ and $f: F \rightarrow \omega^{\omega}$ such that

(i) for any compact $K \subseteq G, g K \subseteq F$ for some $g \in G$, and $f$ is a continuous, open surjection;

(ii) for $A \subseteq \omega^{\omega}, A$ is non-dominating if and only if $f^{-1}(A)$ is Haar null.

We will give a proof of this theorem first and then establish several of its corollaries. For $s \in \omega^{<\omega}$ let $|s|$ be the unique $n \in \omega$ with $s \in \omega^{n}$. Fix an invariant metric $d$ on $G$. Each such metric is complete. For $A, B \subseteq G$ define

$$
\operatorname{dist}(A, B)=\inf \{d(g, h): g \in A, h \in B\} .
$$

We write $r \ll s$, for two nonnegative real numbers $s$ and $r$, if the ball $B(1, s)$ cannot be covered by finitely many balls of radius $r$. Note that $r \ll s$ implies $r \leq s$. Let $\delta$ and $\varepsilon$ be two positive numbers. Define a set $A$ to be $(\delta, \varepsilon)$-discrete if for any $x, y \in A$ either $d(x, y)<\varepsilon$ or $d(x, y)>\delta$. The name $(\delta, \varepsilon)$-discrete is justified by the fact that this definition will be applied in situations in which $\varepsilon$ is much smaller than $\delta$.

The following lemma generalizes the result proved by Dougherty [D, Proposition 12] that each compact subset of a Polish group with an invariant metric is Haar null. To see that this is indeed a generalization of this result note that since each ball of radius $\varepsilon / 2>0$ is $(\delta, \varepsilon)$-discrete for any $\delta>0$, each compact set is a union of finitely many $(\delta, \varepsilon)$-discrete sets for arbitrary $\delta, \varepsilon>0$.

Lemma 2.2. Let $G$ be as in Theorem 2.1. Let $\left(A_{n}\right)$ be a sequence of subsets of $G$ such that each $A_{n}$ is a finite union of $\left(\delta_{n}, \varepsilon_{n}\right)$-discrete sets. If

$$
\sum_{i>n} \delta_{i}<2 \varepsilon_{n} \ll \delta_{n} / 4
$$

then $\bigcap_{m} \bigcup_{n \geq m} A_{n}$ is Haar null.

Proof. Let $A_{n}=\bigcup_{k<k_{n}} B_{k}^{n}$ with each $B_{k}^{n}\left(\delta_{n}, \varepsilon_{n}\right)$-discrete. Find $D_{n} \subseteq$ $B\left(0, \delta_{n} / 4\right)$ so that $\left|D_{n}\right|=2^{n} k_{n}$ and any two distinct elements of $D_{n}$ are at distance not smaller than $2 \varepsilon_{n}$. This can be done since $2 \varepsilon_{n} \ll \delta_{n} / 4$. Consider the compact metric space $\prod_{i} D_{i}$ equipped with a Borel probability measure $\mu$ which is the product measure of the measures $\mu_{i}$ where $\mu_{i}$ assigns the same weight $1 /\left|D_{i}\right|$ to each point in $D_{i}$. Let $\phi: \prod_{i} D_{i} \rightarrow G$ be defined by letting $\phi(x)=\prod_{i} x_{i}$ if $x=\left(x_{0}, x_{1}, \ldots\right)$. The invariance of $d$ and the condition $\sum_{i} \delta_{i}<\infty$ guarantee that $\phi$ is well defined and continuous. Let $\nu$ be the Borel probability measure on $G$ given by $\nu(A)=\mu\left(\phi^{-1}(A)\right)$ for any Borel subset $A$ of $G$. We show that $\nu$ witnesses Haar nullness of $\bigcap_{m} \bigcup_{n \geq m} A_{n}$. 
For $\sigma \in \prod_{i<n} D_{k}$ let $N_{\sigma}$ stand for the clopen subset of $\prod_{i} D_{i}$ of all $x$ with $\sigma=\left(x_{0}, \ldots, x_{n-1}\right)$.

Claim. Let $\sigma \in \prod_{i<n} D_{i}$ and let $g, h \in G$. If $x, y \in \phi^{-1}\left[g B_{k}^{n} h\right] \cap N_{\sigma}$, then $x_{n}=y_{n}$.

Proof. Assume that $x_{n} \neq y_{n}$. Then $\prod_{i} x_{i}, \prod_{i} y_{i} \in g B_{k}^{n} h$ and, by invariance of $d, d\left(\prod_{i} x_{i}, \prod_{i} y_{i}\right)=d\left(x_{n} \prod_{i>n} x_{i}, y_{n} \prod_{i>n} y_{i}\right)$. Thus, again by invariance of $d$,

$$
\begin{aligned}
d\left(\prod_{i} x_{i}, \prod_{i} y_{i}\right) & \leq d\left(x_{n}, y_{n}\right)+d\left(\prod_{i>n} x_{i}, 1\right)+d\left(\prod_{i>n} y_{i}, 1\right) \\
& \leq \delta_{n} / 2+2 \sum_{i>n} \delta_{i} / 4<\delta_{n}
\end{aligned}
$$

and

$$
\begin{aligned}
d\left(\prod_{i} x_{i}, \prod_{i} y_{i}\right) & \geq d\left(x_{n}, y_{n}\right)-d\left(\prod_{i>n} x_{i}, 1\right)-d\left(\prod_{i>n} y_{i}, 1\right) \\
& \leq 2 \varepsilon_{n}-2 \sum_{i>n} \delta_{i} / 4>\varepsilon_{n} .
\end{aligned}
$$

These two inequalities show that $g B_{k}^{n} h$ is not $\left(\delta_{n}, \varepsilon_{n}\right)$-discrete, which, by invariance of $d$, contradicts $\left(\delta_{n}, \varepsilon_{n}\right)$-discreteness of $B_{k}^{n}$. This proves the claim.

Let $\nu^{*}$ and $\mu^{*}$ stand for the outer measures associated with $\nu$ and $\mu$, respectively. From the Claim we deduce that for any $g, h \in G$,

$$
\begin{aligned}
\nu^{*}\left(g B_{k}^{n} h\right) & =\mu^{*}\left(\phi^{-1}\left[g B_{k}^{n} h\right]\right)=\sum_{\sigma \in \prod_{i<n} D_{i}} \mu^{*}\left(\phi^{-1}\left[g B_{k}^{n} h\right] \cap N_{\sigma}\right) \\
& \leq \sum_{\sigma \in \prod_{i<n} D_{i}} \frac{1}{\left|D_{n}\right|} \mu\left(N_{\sigma}\right)=\frac{1}{2^{n} k_{n}} .
\end{aligned}
$$

Thus,

$$
\nu^{*}\left(g A_{n} h\right) \leq \sum_{k<k_{n}} \nu^{*}\left(g B_{k}^{n} h\right) \leq \frac{k_{n}}{2^{n} k_{n}}=2^{-n} .
$$

This implies that $\mu\left(g \bigcap_{m} \bigcup_{n \geq m} A_{n} h\right)=0$ for $g, h \in G$.

Lemma 2.3. Let $G$ be as in Theorem 2.1. Each open non-empty subset of $G$ contains a closed set which is Haar null and homeomorphic to $\omega^{\omega}$.

Proof. Let $U$ be a ball, say of radius $\varepsilon / 2>0$, whose closure is included in the open set in question. Fix two sequences $\left(\delta_{n}\right)$ and $\left(\varepsilon_{n}\right)$ of positive numbers in such a way that for each $n, \sum_{i>n} \delta_{i}<2 \varepsilon_{n} \ll \delta_{n} / 4$ and $\delta_{n}+\varepsilon_{n} \ll \varepsilon_{n-1} / 4$ with $\varepsilon_{-1}=\varepsilon$. Let $d$ be an invariant metric on $G$. By invariance and since $\delta_{n}+\varepsilon_{n} \ll \varepsilon_{n-1} / 4$, each ball of radius $\varepsilon_{n-1} / 4$ contains infinitely many points which are at least $\delta_{n}+\varepsilon_{n}$ apart. Thus, since additionally $\varepsilon_{n-1} / 4>\varepsilon_{n} / 2$, 
each ball $B$ of radius $\varepsilon_{n-1} / 2$ contains closures of infinitely many balls $B_{k}$, $k \in \omega$, of radius $\varepsilon_{n} / 2$ and such that $\operatorname{dist}\left(B_{k}, B_{l}\right)>\delta_{n}$ if $k \neq l$. Thus $\bigcup_{k} B_{k}$ is contained in $B$ and is $\left(\delta_{n}, \varepsilon_{n}\right)$-discrete. Using this observation, construct balls $B_{s}, s \in \omega^{<\omega}$, so that

(1) $B_{\emptyset}=U$;

(2) $B_{s}$ is of radius $\varepsilon_{|s|-1} / 2$;

(3) $\operatorname{dist}\left(B_{s k}, B_{s l}\right) \geq \delta_{|s|}$ if $k \neq l, k, l \in \omega$;

(4) $\bar{B}_{t} \subseteq B_{s}$ if $t \subseteq s$.

Note that $(3)$ and $(4)$ and the fact that $\left(\delta_{n}\right)$ is a decreasing sequence ensure that $\operatorname{dist}\left(B_{s}, B_{t}\right) \geq \delta_{n}$ if $s, t \in \omega^{n+1}$ and $s \neq t$. Thus $\bigcup_{s \in \omega^{n+1}} \bar{B}_{s}$ is closed and, by also (2) and $(3),\left(\delta_{n}, \varepsilon_{n}\right)$-discrete. It follows that $H=$ $\bigcap_{n} \bigcup_{s \in \omega^{n+1}} \bar{B}_{s}$ is closed and by Lemma 2.2 Haar null. It is routine to check that the function $g: \omega^{\omega} \rightarrow H$ defined by letting $g(x)$ be the unique point in $\bigcap_{n} \bar{B}_{x \mid n}$ is a homeomorphism.

Proof of Theorem 2.1. Let $\left(Q_{k}\right)$ be a sequence of finite subsets of $G$ such that $1 \in Q_{0}, Q_{k} \subseteq Q_{k+1}$, and $\bigcup_{k} Q_{k}$ dense in $G$. Fix now three sequences $\left(\varepsilon_{n}\right),\left(\delta_{n}\right)$ and $\left(r_{n}\right)$ of positive numbers in such a way that for each $n$,

$$
r_{n} \gg 5 \delta_{n}, \quad \delta_{n} / 4 \gg 8 \varepsilon_{n}, \quad \text { and } \quad \varepsilon_{n}>\sum_{k>n} r_{k} .
$$

Since the group is not locally compact, for any positive number $a$ there exists $b>0$ such that $a \gg b$. Thus, the choice of $r_{n}, \delta_{n}$ and $\varepsilon_{n}$ is possible.

Now for each $n$ fix a sequence $\left(g_{k}^{n}\right)_{k}$ so that

$$
g_{k}^{n} \in B\left(1, r_{n}\right) \quad \text { and } \quad \operatorname{dist}\left(g_{k}^{n} Q_{k}, \bigcup_{i<k} g_{i}^{n} Q_{i}\right) \geq 5 \delta_{n} .
$$

It requires finding for each $k$ a $g_{k}^{n} \in B\left(1, r_{n}\right)$ such that

$$
\operatorname{dist}\left(g_{k}^{n}, \bigcup_{i<k} g_{i}^{n} Q_{i} Q_{k}^{-1}\right) \geq 5 \delta_{n}
$$

and this can be done since $5 \delta_{n} \ll r_{n}$.

Let $V_{k}^{n}=B\left(1, \varepsilon_{n}\right) Q_{k}$ and $U_{k}^{n}=B\left(0,2 \varepsilon_{n}\right) Q_{k}$. We will need the following two claims.

Claim 1. If $s, t \in \omega^{n+1}$ and $s \neq t$, then each point in

$$
g_{s(0)}^{0} \overline{U_{s(0)}^{0}} \cap g_{s(0)}^{0} g_{s(1)}^{1} \overline{U_{s(1)}^{1}} \cap \ldots \cap g_{s(0)}^{0} g_{s(1)}^{1} \ldots g_{s(n)}^{n} \overline{U_{s(n)}^{n}}
$$

is at distance greater than $2 \delta_{n}$ from any point of

$$
g_{t(0)}^{0} \overline{U_{t(0)}^{0}} \cap g_{t(0)}^{0} g_{t(1)}^{1} \overline{U_{t(1)}^{1}} \cap \ldots \cap g_{t(0)}^{0} g_{t(1)}^{1} \ldots g_{t(n)}^{n} \overline{U_{t(n)}^{n}} .
$$


Proof. Let $n_{0} \leq n$ be the smallest natural number such that $s\left(n_{0}\right) \neq$ $t\left(n_{0}\right)$. In particular, $s\left|n_{0}=t\right| n_{0}$. It will suffice to show that

$$
\operatorname{dist}\left(g_{s(0)}^{0} \ldots g_{s\left(n_{0}-1\right)}^{n_{0}-1} g_{s\left(n_{0}\right)}^{n_{0}} \overline{U_{s\left(n_{0}\right)}^{n_{0}}}, g_{s(0)}^{0} \ldots g_{s\left(n_{0}-1\right)}^{n_{0}-1} g_{t\left(n_{0}\right)}^{n_{0}} \overline{U_{t\left(n_{0}\right)}^{n_{0}}}\right)>2 \delta_{n},
$$

which by invariance of the metric amounts to noticing that

$$
\operatorname{dist}\left(g_{s\left(n_{0}\right)}^{n_{0}} \overline{U_{s\left(n_{0}\right)}^{n_{0}}}, g_{t\left(n_{0}\right)}^{n_{0}} \overline{\left.U_{t\left(n_{0}\right)}^{n_{0}}\right)}>2 \delta_{n} .\right.
$$

This is true by invariance of the metric and the facts that $\operatorname{dist}\left(g_{s\left(n_{0}\right)}^{n_{0}} Q_{s\left(n_{0}\right)}\right.$, $\left.g_{t\left(n_{0}\right)}^{n_{0}} Q_{t\left(n_{0}\right)}\right) \geq 5 \delta_{n}, U_{s\left(n_{0}\right)}^{n_{0}}=B\left(1,2 \varepsilon_{n}\right) Q_{s\left(n_{0}\right)}$ and $U_{t\left(n_{0}\right)}^{n_{0}}=B\left(1,2 \varepsilon_{n}\right) Q_{t\left(n_{0}\right)}$, and $2 \varepsilon_{n} \leq \delta_{n}$. Thus, the claim is established.

Claim 2. For each $n, k$ and any choice of $\left(k_{i}\right) \in \omega^{\omega}, \prod_{i>n} g_{k_{i}}^{i} V_{k}^{n}$ $\subseteq U_{k}^{n}$.

Proof. By invariance of $d$,

$$
d\left(1, \prod_{i>n} g_{k_{i}}^{i}\right) \leq \sum_{i>n} d\left(1, g_{k_{i}}^{i}\right) \leq \sum_{i>n} r_{i}<\varepsilon_{n} .
$$

This implies that for any $q \in Q_{k}, \prod_{i>n} g_{k_{i}}^{i} B\left(1, \varepsilon_{n}\right) q \subseteq B\left(1,2 \varepsilon_{n}\right) q$. This leads directly to the conclusion of the claim.

Define

$$
F_{1}=\bigcap_{n} \bigcup_{s \in \omega^{n+1}} g_{s(0)}^{0} \overline{U_{s(0)}^{0}} \cap g_{s(0)}^{0} g_{s(1)}^{1} \overline{U_{s(1)}^{1}} \cap \ldots \cap g_{s(0)}^{0} g_{s(1)}^{1} \ldots g_{s(n)}^{n} \overline{U_{s(n)}^{n}} .
$$

The following two properties of $F_{1}$ will be needed later:

(1) $F_{1}$ is closed;

(2) $F_{1}$ is nowhere dense.

We show (1) first. Note that

$$
g_{s(0)}^{0} \overline{U_{s(0)}^{0}} \cap g_{s(0)}^{0} g_{s(1)}^{1} \overline{U_{s(1)}^{1}} \cap \ldots \cap g_{s(0)}^{0} g_{s(1)}^{1} \ldots g_{s(n)}^{n} \overline{U_{s(n)}^{n}}
$$

is closed for any $s \in \omega^{n+1}$. By Claim 1 ,

$$
\bigcup_{s \in \omega^{n+1}} g_{s(0)}^{0} \overline{U_{s(0)}^{0}} \cap g_{s(0)}^{0} g_{s(1)}^{1} \overline{U_{s(1)}^{1}} \cap \ldots \cap g_{s(0)}^{0} g_{s(1)}^{1} \ldots g_{s(n)}^{n} \overline{U_{s(n)}^{n}}
$$

is closed as well, whence $F_{1}$ is closed being the intersection of closed sets.

Having proved that $F_{1}$ is closed, proving (2) requires only checking that $F_{1}$ has empty interior. If not, then for some $\delta_{n}$ a ball of radius $\delta_{n}$ is contained in $F_{1}$. By the definition of $F_{1}$, this ball would be included in

$$
\bigcup_{s \in \omega^{n+1}} g_{s(0)}^{0} \overline{U_{s(0)}^{0}} \cap g_{s(0)}^{0} g_{s(1)}^{1} \overline{U_{s(1)}^{1}} \cap \ldots \cap g_{s(0)}^{0} g_{s(1)}^{1} \ldots g_{s(n)}^{n} \overline{U_{s(n)}^{n}} .
$$

By Claim 1, this implies that the ball is included in

$$
g_{s(0)}^{0} g_{s(1)}^{1} \ldots g_{s(n)}^{n} \overline{U_{s(n)}^{n}}
$$


for some $s \in \omega^{n+1}$. But this is not possible since this set can be covered by finitely many balls of radius $\leq 2 \varepsilon_{n}$ and this cannot be done with a ball of radius $\delta_{n}$ as $2 \varepsilon_{n} \ll \delta_{n}$. So (2) is established as well.

Define, for $x \in \omega^{\omega}$,

$$
K_{x}=\bigcap_{n} g_{x(0)}^{0} \ldots g_{x(n)}^{n} \overline{U_{x(n)}^{n}} .
$$

We register now three properties of the family of all $K_{x}$ 's for future use:

(3) for each $x \in \omega^{\omega}, \prod_{n} g_{x(n)}^{n} \in \bigcap_{n} g_{x(0)}^{0} \ldots g_{x(n)}^{n} U_{x(n)}^{n}$;

(4) if $x \neq y$, then $K_{x} \cap K_{y}=\emptyset$;

(5) $F_{1}=\bigcup_{x \in \omega^{\omega}} K_{x}$.

To prove (3) note that by Claim 2 ,

$$
\prod_{n} g_{x(n)}^{n}=g_{x(0)}^{0} \ldots g_{x(m)}^{m} \prod_{n>m} g_{x(n)}^{n} \in g_{x(0)}^{0} \ldots g_{x(m)}^{m} U_{x(m)}^{m} .
$$

Since this happens for each $m, \prod_{n} g_{x(n)}^{n} \in \bigcap_{n} g_{x(0)}^{0} \ldots g_{x(m)}^{m} U_{x(m)}^{m}$.

To see (4), fix $x, y \in \omega^{\omega}$ with $x \neq y$. Let $n_{0}$ be the smallest natural number with $x\left(n_{0}\right) \neq y\left(n_{0}\right)$. Then

$$
\begin{aligned}
& K_{x} \subseteq g_{x(0)}^{0} \overline{U_{x(0)}^{0}} \cap \ldots \cap g_{x(0)}^{0} \ldots g_{x\left(n_{0}-1\right)}^{n_{0}-1} g_{x\left(n_{0}\right)}^{n_{0}} \overline{U_{x\left(n_{0}\right)}^{n_{0}}}, \\
& K_{y} \subseteq g_{y(0)}^{0} \overline{U_{y(0)}^{0}} \cap \ldots \cap g_{y(0)}^{0} \ldots g_{y\left(n_{0}-1\right)}^{n_{0}-1} g_{y\left(n_{0}\right)}^{n_{0}} \overline{U_{y\left(n_{0}\right)}^{n_{0}}},
\end{aligned}
$$

and disjointness of $K_{x}$ and $K_{y}$ follows from Claim 1.

Now an argument for (5). For any $x, K_{x} \subseteq F_{1}$ is completely clear, so $\bigcup_{x \in \omega^{\omega}} K_{x} \subseteq F_{1}$. Let $z \in F_{1}$. Then, by Claim 1 , for each $n$ there is a unique $s_{n} \in \omega^{n+1}$ such that

$$
z \in g_{s_{n}(0)}^{0} \overline{U_{s_{n}(0)}^{0}} \cap \ldots \cap g_{s_{n}(0)}^{0} \ldots g_{s_{n}(n)}^{n} \overline{U_{s_{n}(n)}^{n}} .
$$

It follows that $s_{n} \subseteq s_{n+1}$. Then for $x=\bigcup_{n} s_{n}$, we obviously have $z \in K_{x}$.

Now fix sets $H_{i}^{s}, s \in \omega^{<\omega}, i \leq i_{s}$ for some finite $i_{s}$, so that

(6) $H_{i}^{s} \subseteq\left(g_{s(0)}^{0} U_{s(0)}^{0} \cap \ldots \cap g_{s(0)}^{0} g_{s(1)}^{1} \ldots g_{s(n)}^{n} U_{s(n)}^{n}\right) \backslash F_{1}$;

(7) $H_{i}^{s}$ closed and homeomorphic to $\omega^{\omega}$;

(8) $H_{i}^{s}$ Haar null;

(9) the diameter of $H_{i}^{s}$ is less than $1 /(|s|+1)$;

(10) each point in $g_{s(0)}^{0} U_{s(0)}^{0} \cap \ldots \cap g_{s(0)}^{0} g_{s(1)}^{1} \ldots g_{s(n)}^{n} U_{s(n)}^{n}$ is at distance not greater than $5 \varepsilon_{n}$ from $H_{i}^{s}$;

(11) $H_{i}^{s} \cap H_{j}^{t}=\emptyset$ if $i \neq j$ or $s \neq t$.

Here is why such a choice of $i_{s}$ and $H_{i}^{s}, i \leq i_{s}$, is possible. Let $\left(s_{n}\right)$ be an enumeration of $\omega^{<\omega}$. Assume $i_{s_{k}}$ and $H_{i}^{s_{k}}$ for $i \leq i_{s_{k}}$ have been defined for $k<n$. Let $s_{n}=s$. Note first that the set $g_{s(0)}^{0} U_{s(0)}^{0} \cap \ldots \cap$ $g_{s(0)}^{0} g_{s(1)}^{1} \ldots g_{s(n)}^{n} U_{s(n)}^{n}$ is included in $g_{s(0)}^{0} g_{s(1)}^{1} \ldots g_{s(n)}^{n} U_{s(n)}^{n}$, which is the 
union of finitely many, say $i_{s}$ many, sets of diameter not larger than $4 \varepsilon_{n}$. Thus, we can pick $i_{s}$ points in it so that each point of it is at distance not more than $4 \varepsilon_{n}$ from one of the finitely many points picked. The set

$$
\left(g_{s(0)}^{0} U_{s(0)}^{0} \cap \ldots \cap g_{s(0)}^{0} g_{s(1)}^{1} \ldots g_{s(n)}^{n} U_{s(n)}^{n}\right) \backslash\left(F_{1} \cup \bigcup_{k<n} \bigcup_{i \leq i_{s_{k}}} H_{i}^{s_{k}}\right)
$$

is open and non-empty since, by (4),

$$
g_{s(0)}^{0} U_{s(0)}^{0} \cap \ldots \cap g_{s(0)}^{0} g_{s(1)}^{1} \ldots g_{s(n)}^{n} U_{s(n)}^{n} \neq \emptyset
$$

and, by (1) and (2), $F_{1}$ is closed and nowhere dense, as are the sets $H_{i}^{s_{k}}$ since, by our inductive assumption, they are closed and Haar null. Now, it follows from Lemma 2.3 that in any neighborhood of the $i$ th point out of the $i_{s}$ points chosen we can find a closed copy of $\omega^{\omega}$ which is Haar null. This allows us to define $H_{i}^{s}$ for $i \leq i_{s}$ so that (6)-(11) are satisfied.

Define now

$$
F=F_{1} \cup \bigcup_{s \in \omega<\omega} \bigcup_{i \leq i_{s}} H_{i}^{s} .
$$

We show that $F$ is closed. Since $F_{1}$ is closed and all the $H_{i}^{s}$ 's are closed as well, it will suffice to show that any convergent sequence $\left(x_{n}\right)$ contained in $\bigcup_{s \in \omega<\omega} \bigcup_{i \leq i_{s}} H_{i}^{s}$ which has only finitely many points in each $H_{i}^{s}$ converges to a point in $F_{1}$. Using Claim 1, property (6) of sets $H_{i}^{s}$, and $i_{s}<\infty$, we see that we can pass to a subsequence of the sequence $\left(x_{n}\right)$, which we again call $\left(x_{n}\right)$, for which there exist $s_{n} \in \omega^{n+1}, n \in \omega$, with $s_{n} \subseteq s_{n+1}$ and for for each $n$ and $m \geq n$,

$$
x_{m} \in g_{s_{n}(0)}^{0} \overline{U_{s_{n}(0)}^{0}} \cap \ldots \cap g_{s_{n}(0)}^{0} \ldots g_{s_{n}(n)}^{n} \overline{U_{s_{n}(n)}^{n}} .
$$

Since the sets $g_{s_{n}(0)}^{0} \overline{U_{s_{n}(0)}^{0}} \cap \ldots \cap g_{s_{n}(0)}^{0} \ldots g_{s_{n}(n)}^{n} \overline{U_{s_{n}(n)}^{n}}$ are closed, $\lim _{n} x_{n}$ belongs to all of them, so also to their intersection, which is $K_{z}$, for $z=$ $\bigcup_{n} s_{n}$, and this set is included in $F_{1}$ by (5).

Fix now homeomorphisms $\phi_{i}^{s}: H_{i}^{s} \rightarrow \omega^{\omega}$. Finally define $f: F \rightarrow \omega^{\omega}$ as follows. If $z \in F_{1}$, let $f(z)$ be the unique $x \in \omega^{\omega}$ with $z \in K_{x}$. If $z \in H_{i}^{s}$ let $f(z)=s \phi_{i}^{s}(z)$. The function $f$ is well defined by (3) and (5).

We will now check (i) from the conclusion of the theorem. We first show that for any compact set $K$ there exists a $g \in G$ with $g K \subseteq F$. Actually, we will have $g K \subseteq F_{1}$. For each $i, \bigcup_{k} V_{k}^{i}=G$ since $\bigcup_{k} Q_{k}$ is dense in $G$. This and compactness of $K$ allow us to pick a sequence $\left(k_{i}\right)$ so that $K \subseteq V_{k_{i}}^{i}$. By Claim 2, for each $n$,

$$
\left(\prod_{i} g_{k_{i}}^{i}\right) K \subseteq\left(\prod_{i \leq n} g_{k_{i}}^{i}\right)\left(\prod_{i>n} g_{k_{i}}^{i}\right) V_{k_{n}}^{n} \subseteq\left(\prod_{i \leq n} g_{k_{i}}^{i}\right) U_{k_{n}}^{n}
$$

Thus, $\left(\prod_{i} g_{k_{i}}^{i}\right) K \subseteq K_{\left(k_{i}\right)} \subseteq F_{1}$. 
The very definition of $f$ insures that it is a surjection. Now we show that $f$ is continuous and open. First continuity. Fix a converging sequence $\left(x_{n}\right) \in F$ with limit $x$. Eliminating the obvious possibilities and passing to a subsequence, we can assume that $x \in F_{1}$ and we have two cases.

Case 1: $\left(x_{n}\right)$ included in $\bigcup_{s \in \omega<\omega} \bigcup_{i \leq i_{s}} H_{i}^{s}$. Again, as in the proof that $F$ is closed, by passing to a subsequence, we can produce a sequence $s_{n} \in$ $\omega^{n+1}$ with $s_{n} \subseteq s_{n+1}$ and for $m \geq n$,

$$
x_{m} \in g_{s_{n}(0)}^{0} \overline{U_{s_{n}(0)}^{0}} \cap \ldots \cap g_{s_{n}(0)}^{0} \ldots g_{s_{n}(n)}^{n} \overline{U_{s_{n}(n)}^{n}} .
$$

Set $z=\bigcup_{n} s_{n}$. By the definition of $f, \lim _{n} f\left(x_{n}\right)=z$. On the other hand, $x \in K_{z}$, so $f(x)=z$.

Case 2: $\left(x_{n}\right)$ included in $F_{1}$. The proof is similar to that in Case 1.

We now check that $f$ is open. Let $U$ be an open subset of $G$ and let $x_{0} \in$ $F \cap U$. We need to find an open set $V \subseteq \omega^{\omega}$ such that $f\left(x_{0}\right) \in V \subseteq f[F \cap U]$. For $s \in \omega^{<\omega}$, let $N_{s}=\left\{z \in \omega^{\omega}: z|| s \mid=s\right\}$. If $x_{0} \in H_{i}^{s}$ for some $s \in \omega^{<\omega}$ and $i \leq i_{s}$, there exists a $t \in \omega^{<\omega}$ such that $x_{0} \in\left(\phi_{i}^{s}\right)^{-1}\left(N_{t}\right) \subseteq U \cap F$. Thus $f\left(x_{0}\right) \in N_{s t}$ and $N_{s t} \subseteq f[U \cap F]$. Assume now $x_{0} \in F_{1}$. By (5) we can find some $y \in \omega^{\omega}$ with $x_{0} \in K_{y}$. Then, by (9) and (10), taking into account the definition of $K_{y}$ and the fact that $\varepsilon_{n}$ tends to 0 , for some $s \subseteq y$ we have $U \cap F \supseteq H_{i}^{s}$. It follows that $f[U \cap F] \supseteq N_{s}$ and $f\left(x_{0}\right)=y \in N_{s}$.

Now we check that (ii) is satisfied. We first show that if $H \subseteq \omega^{\omega}$ is dominating, then $A=f^{-1}(H)$ is not Haar null. Fix a Borel probability measure $\mu$ on $G$. By regularity of $\mu$, we can find a compact set $K$ such that $\mu(K)>0$. By subtracting from $K$ all relatively open (in $K$ ) sets of $\mu$ measure zero, we can assume that for all $\emptyset \neq U \subseteq K$ open in $K, \mu(U)>0$. Moreover, without loss of generality, we can assume that $1 \in K$. Pick a sequence $\left(k_{n}\right) \in \omega^{\omega}$ so that for each $n, K \subseteq V_{k_{n}}^{n}$. Find $y \in H$ so that for some $n_{0}, k_{n} \leq y(n)$ for all $n \geq n_{0}$. Note that for $n \geq n_{0}, K \subseteq V_{k_{n}}^{n} \subseteq V_{y(n)}^{n}$. Therefore, for $n \geq n_{0}$, by Claim 2,

$$
\prod_{i} g_{y(i)}^{i} K \subseteq \prod_{i} g_{y(i)}^{i} V_{y(n)}^{n}=\prod_{i \leq n} g_{y(i)}^{i} \prod_{n<i} g_{y(i)}^{i} V_{y(n)}^{n} \subseteq \prod_{i \leq n} g_{y(i)}^{i} \overline{U_{y(n)}^{n}} .
$$

It follows from this and the definition of $K_{y}$ that

$$
\begin{aligned}
\left(\prod_{i} g_{y(i)}^{i} K\right) & \cap K_{y} \\
& \supseteq\left(\prod_{i} g_{y(i)}^{i} K\right) \cap \bigcap_{n \geq n_{0}} \prod_{i \leq n} g_{y(i)}^{i} \overline{U_{y(n)}^{n}} \cap \bigcap_{n<n_{0}} \prod_{i \leq n} g_{y(i)}^{i} U_{y(n)}^{n} \\
& =\left(\prod_{i} g_{y(i)}^{i} K\right) \cap \bigcap_{n<n_{0}} \prod_{i} g_{y(i)}^{i} U_{y(n)}^{n} .
\end{aligned}
$$


This last set is non-empty since $\prod_{i} g_{y(i)}^{i}$ belongs to the two sets which are being intersected by (3) and the fact that $1 \in K$. It is also relatively open in $\prod_{i} g_{y(i)}^{i} K$. It follows that $\mu\left(\left(\prod_{i} g_{y(i)}^{i}\right)^{-1} K_{y}\right)>0$, so $\mu_{*}\left(\left(\prod_{i} g_{y(i)}^{i}\right)^{-1} f^{-1}(H)\right)$ $>0$ where $\mu_{*}$ stands for the inner measure associated with $\mu$.

Now let $H \subseteq \omega^{\omega}$ be non-dominating. Fix $x \in \omega^{\omega}$ with the property that for each $y \in H, y(n) \leq x(n)$ for infinitely many $n$ 's. For $s \in \omega^{n}$, let

$$
B_{s}=\bigcap_{l<n}\left(g_{s(0)}^{0} \ldots g_{s(l)}^{l} \overline{U_{s(l)}^{l}}\right) \cap \bigcup_{m \leq x(n)}\left(g_{s(0)}^{0} \ldots g_{s(n-1)}^{n-1} g_{m}^{n} \overline{U_{m}^{n}}\right) .
$$

Now $B_{s}$ is covered by $\bigcup_{m \leq x(n)} g_{s(0)}^{0} \ldots g_{s(n-1)}^{n-1} g_{m}^{n} \overline{U_{m}^{n}}$ and each set $\overline{U_{m}^{n}}$ is the union of $\left|Q_{m}\right|$ many sets of diameter $\leq 4 \varepsilon_{n}$. So $B_{s}$ can be covered by $\sum_{m \leq x(n)}\left|Q_{m}\right|$ many sets of diameter $\leq 4 \varepsilon_{n}$. For $n \geq 1$, if $s_{1}, s_{2} \in \omega^{n}$ and $s_{1} \neq s_{2}$, then by Claim $1, \operatorname{dist}\left(B_{s_{1}}, B_{s_{2}}\right) \geq 2 \delta_{n-1}>\delta_{n}$. Thus, $\bigcup_{s \in \omega^{n}} B_{s}$ is the union of finitely many (namely $\left.\sum_{m \leq x(n)}\left|Q_{m}\right|\right)\left(\delta_{n}, 4 \varepsilon_{n}\right)$-discrete sets. Since $\sum_{k>n} \delta_{k}<8 \varepsilon_{n}$ (actually, $\sum_{k>n} \delta_{k} \leq \sum_{k>n} r_{k}<\varepsilon_{n}$ ) and $8 \varepsilon_{n} \ll \delta_{n} / 4$, it follows by Lemma 2.2 that $\bigcap_{m} \bigcup_{n \geq m}\left(\bigcup_{s \in \omega^{n}} B_{s}\right)$ is Haar null. If, for some $n, y(n) \leq x(n)$, then

$$
K_{y} \subseteq B_{y \mid n} \subseteq \bigcup_{s \in \omega^{n}} B_{s} .
$$

Now by the choice of $x$, for any $y \in H, K_{y} \subseteq \bigcup_{s \in \omega^{n}} B_{s}$ for infinitely many $n$ 's, whence

$$
K_{y} \subseteq \bigcap_{m} \bigcup_{n \geq m}\left(\bigcup_{s \in \omega^{n}} B_{s}\right)
$$

Since $y$ is an arbitrary member of $H$, it follows that

$$
f^{-1}(H) \subseteq \bigcap \bigcup_{m}\left(\bigcup_{s \in \omega^{n}} B_{s}\right) \cup \bigcup_{s \in \omega<\omega} \bigcup_{i \leq i_{s}} H_{i}^{s} .
$$

Since a countable union of Haar null sets is Haar null, the set on the right hand side of the inclusion is Haar null. Hence $f^{-1}(H)$ is Haar null being a subset of a Haar null set.

REMARK. As we will see the properties of $F$ and $f$ as stated in Theorem 2.1 are sufficient for applications that arose so far. We would like, however, to point out some further properties of $F$ and $f$ as well as state more refined versions of the properties from Theorem 2.1 in case they become useful in some future applications. We will need a couple definitions.

Recall that a continuous function is perfect if preimages of compact sets are compact. Call a subset $A$ of a Polish group $G$ openly Haar null if there exists a probability Borel measure $\mu$ on $G$ such that for any $\varepsilon>0$ we can find an open set $U \subseteq G$ with $A \subseteq U$ and $\mu(g U h)<\varepsilon$ for any $g, h \in G$. Clearly, each openly Haar null set is Haar null. Also, the method of proving that Haar null sets constitute a $\sigma$-ideal can be easily adapted to show that 
openly Haar null sets form a $\sigma$-ideal. I do not know, however, if each Haar null set is openly Haar null. Note that each openly Haar null set is contained in a $G_{\delta}$ set which is Haar null. The only known similar property of Haar null sets is that each analytic Haar null set is contained in a Borel Haar null set [S]. But no estimate on the complexity of the Borel set has been established. On the other hand, methods of Matouškova's paper [M2, Lemma 1.2 and Theorem 1.3] show that if $G$ is a separable Banach space and $A \subseteq G$ is weakly compact, then $A$ is Haar null iff $A$ is openly Haar null.

Now, I will list the additional properties of $f$ and $F$ from Theorem 2.1. I will leave their proofs to the reader as these proofs are only minor modifications of arguments in Theorem 2.1. Let me only mention that point 2 follows from the fact that the sets in Lemma 2.1 are openly Haar null.

1. $f$ is perfect.

Since perfect mappings between metric spaces are closed (see [E]), openness, continuity, and perfectness of $f$ give that preimages and images of closed, open, and compact sets are closed, open and compact, respectively.

2. If $H \subseteq \omega^{\omega}$ is non-dominating, then $f^{-1}(H)$ is openly Haar null.

3. If $K \subseteq G$ is compact, then $g K \subseteq f^{-1}(x)$ for some $x \in \omega^{\omega}$ and some $g \in G$.

4. For $x, y \in \omega^{\omega}$, if $x \leq^{*} y$, then for some countable $D \subseteq G, f^{-1}(x) \subseteq$ $D f^{-1}(y)$.

5. If $H \subseteq \omega^{\omega}$ is dominating and $K \subseteq G$ is compact, then for some $g_{n} \in G$ and compact $K_{n} \subseteq K$ with $K=\bigcup_{n} K_{n}, n \in \omega$, we have $\bigcup_{n} g_{n} K_{n} \subseteq$ $f^{-1}(H)$.

3. Complexity of closed Haar null sets and other applications. For a Polish space $X$ let $\mathcal{F}(X)$ be the family of all closed subsets of $X$. Let $\mathcal{F}(X)$ be equipped with Fell's topology whose subbasis is constituted by two kinds of subsets of $\mathcal{F}(X)$ : first, all closed subsets of $X$ disjoint from a given compact subset of $X$, second, all closed subsets of $X$ intersecting a given open subset of $X$. As is easy to see, the Borel sets of this topology coincide with the Borel structure generated by sets of the form $\{F \in \mathcal{F}(X): F \cap U$ $\neq \emptyset\}$ for some open set $U \subseteq X$. This Borel structure is standard, that is, is isomorphic to the Borel structure of a Polish space and is called the Effros Borel structure (see $[\mathrm{K}, 12.6]$ ).

By CD we denote the family of all closed dominating subsets of $\omega^{\omega}$ and by CND the family of all closed non-dominating subsets of $\omega^{\omega}$. We equip $\mathcal{F}(G)$ and $\mathcal{F}\left(\omega^{\omega}\right)$ with Fell's topology.

Lemma 3.1. Let $G$ be a Polish, non-locally compact group with an invariant metric. There exists a continuous function $\Phi: \mathcal{F}\left(\omega^{\omega}\right) \rightarrow \mathcal{F}(G)$ such 
that for $H \in \mathcal{F}\left(\omega^{\omega}\right)$,

$$
H \in \mathrm{CND} \text { iff } \Phi(H) \text { is Haar null. }
$$

Proof. Let $F$ and $f: F \rightarrow \omega^{\omega}$ be as in Theorem 2.1. Define $\Phi: \mathcal{F}\left(\omega^{\omega}\right) \rightarrow$ $\mathcal{F}(G)$ by

$$
\Phi(H)=f^{-1}(H) .
$$

Since $f$ is continuous, $f^{-1}(H)$ is closed if $H$ is, so $\Phi$ is well defined. Now, $H$ is not dominating if and only if $\Phi(H)$ is Haar null by Theorem 2.1.

It remains to see that $\Phi$ is continuous, which amounts to showing that for any open $V \subseteq G$ and compact $L \subseteq G$ the preimages of the sets

$$
\{H \in \mathcal{F}(G): H \cap V \neq \emptyset\} \quad \text { and } \quad\{H \in \mathcal{F}(G): H \cap L=\emptyset\}
$$

are open. A short calculation shows that the preimages are, respectively,

$$
\left\{H \in \mathcal{F}\left(\omega^{\omega}\right): H \cap f[V] \neq \emptyset\right\} \quad \text { and } \quad\left\{H \in \mathcal{F}\left(\omega^{\omega}\right): H \cap f[L]=\emptyset\right\} .
$$

These sets are open in $\mathcal{F}\left(\omega^{\omega}\right)$ since $f$ is open and continuous.

A pair of subsets $(A, B)$ of a Polish space $X$ will be called $\boldsymbol{\Pi}_{1}^{1}$-hard if for any $\Pi_{1}^{1}$ subset $C$ of a Polish space $Y$ there exists a Borel function $f: Y \rightarrow X$ such that $f(x) \in A$ for $x \in C$ and $f(x) \in B$ for $x \in Y \backslash C$. A subset $A$ of a Polish space $X$ is called $\Pi_{1}^{1}$-hard if the pair $(A, X \backslash A)$ is $\Pi_{1}^{1}$-hard. The following lemma contains a bit more than we will need in applications.

LEMmA 3.2. (i) The pair $(\{H \in \mathcal{F}: H$ is countable $\}, C D)$ is $\Pi_{1}^{1}$-hard.

(ii) CND is $\boldsymbol{\Pi}_{1}^{1}$-hard, so in particular, it is not $\boldsymbol{\Sigma}_{1}^{1}$ hence not Borel.

Proof. By PTr we denote the family of all pruned trees on $\omega$, that is, $T \in \mathrm{P} T r$ precisely when

$$
\forall s \in T \forall n<|s| \quad s \mid n \in T \quad \text { and } \quad \forall s \in T \exists n \in \omega \quad s n \in T .
$$

Thus PTr is a $G_{\delta}$ subset of the metric compact space $2^{\omega<\omega}$. The mapping $T \mapsto[T]=\left\{x \in \omega^{\omega}: \forall n x \mid n \in T\right\}$ establishes a 1-to-1 correspondence between $\mathrm{PTr}$ and $\mathcal{F}\left(\omega^{\omega}\right)$. It is not difficult to check that this mapping is a Borel isomorphism between PTr with its family of Borel sets and $\mathcal{F}\left(\omega^{\omega}\right)$ with the Effros Borel structure. So it suffices to show that the pair $(\{T \in$ $\mathrm{PTr}:[T]$ countable $\},\{T \in \mathrm{P} \operatorname{Tr}:[T]$ dominating $\})$ is $\boldsymbol{\Pi}_{1}^{1}$-hard. The mapping $\phi: 2^{\omega} \rightarrow \mathrm{PTr}$ given by

$$
\phi(\alpha)=\left\{s \in \omega^{<\omega}: \forall n>0(n<|s| \text { and } \alpha(n)=0 \Rightarrow s(n-1)=s(n))\right\}
$$

is easily checked to be continuous. (Clearly, $\phi(\alpha)$ is a pruned tree on $\omega$, so $\phi$ is well defined.) Let $Q \subseteq 2^{\omega}$ consist of all sequences $\alpha \in 2^{\omega}$ which are eventually 0 . We claim that

$\alpha \in Q \Rightarrow[\phi(\alpha)]$ is countable and $\alpha \notin Q \Rightarrow[\phi(\alpha)]$ is dominating. 
Let $\alpha \in Q$, say $\alpha(n)=0$ for $n>n_{0}$. Then for all $x \in[\phi(\alpha)], x(n)=x\left(n_{0}\right)$ for all $n>n_{0}$. Thus, $[\phi(\alpha)]$ is countable. Let now $\alpha \notin Q$. Put $\left\{n_{0}<n_{1}\right.$ $<\ldots\}=\{n: \alpha(n)=1\}$. Fix $x \in \omega^{\omega}$ and define $y \in \omega^{\omega}$ by letting

$$
y(k)= \begin{cases}0 & \text { if } k<n_{0}, \\ 1+\max \left\{x(i): i<n_{j+1}\right\} & \text { if } n_{j} \leq k<n_{j+1} .\end{cases}
$$

Then it is easy to check that $x \leq^{*} y$ and $y \in[\phi(\alpha)]$. Since $x$ was arbitrary, this shows that $[\phi(\alpha)]$ is dominating.

Now we will use a trick from $[\mathrm{KLW}]$ to finish off the proof. By $\mathcal{K}\left(2^{\omega}\right)$ we denote the space of all compact subsets of $2^{\omega}$ topologized using the Hausdorff metric. Define $\Phi: \mathcal{K}\left(2^{\omega}\right) \rightarrow \mathrm{P} \operatorname{Tr}$ by

$$
\Phi(K)=\bigcup\{\phi(\alpha): \alpha \in K\} .
$$

Note that the union of pruned trees is a pruned tree, so $\Phi$ is well defined. Moreover, for $s \in \omega^{<\omega}, s \in \Phi(K)$ iff $\exists \alpha \in K s \in \phi(\alpha)$, which is a closed condition on $K$. Thus, $\Phi$ is Borel. A short calculation shows that if $K$ is compact, then $[\bigcup\{\phi(\alpha): \alpha \in K\}]=\bigcup\{[\phi(\alpha)]: \alpha \in K\}$. Hence if $K \subseteq Q$, then $[\Phi(K)]$ is countable. If, on the other hand, $K \nsubseteq Q$, then we can fix $\alpha_{0} \in K \backslash Q$, and $[\Phi(K)]$ is dominating as it contains a dominating set $\phi\left(\alpha_{0}\right)$. As $\left\{K \in \mathcal{K}\left(2^{\omega}\right): K \subseteq Q\right\}$ is $\Pi_{1}^{1}$-hard by Mazurkiewicz's theorem (see $[\mathrm{K}]$ ), we are done with (i); (ii) follows immediately from (i).

Remark. Greg Hjorth $[\mathrm{H}]$ showed recently that CND is $\boldsymbol{\Sigma}_{1}^{1}$-hard, as well. On the other hand, it follows from the work of Brendle, Hjorth and Spinas [BHS] that CND is $\boldsymbol{\Delta}_{2}^{1}$. Let me sketch an argument for this last estimate. As $H \subseteq \omega^{\omega}$ is non-dominating precisely when $\exists x \in \omega^{\omega} \forall y \in F$ $y(n) \leq x(n)$ for infinitely many $n$ 's, CND is $\boldsymbol{\Sigma}_{2}^{1}$. To see that it is also $\boldsymbol{\Pi}_{2}^{1}$ recall from [BHS] the definition of nice sets. Consider first the family $S$ of all sequences $\left(\left(w_{\sigma}, s_{\sigma}\right): \sigma \in \omega^{<\omega}\right)$ with the following properties: $w_{\sigma} \subseteq \omega$ is finite, $\operatorname{dom}\left(s_{\emptyset}\right) \subseteq \omega$ is finite and $s_{\emptyset}: \operatorname{dom}\left(s_{\emptyset}\right) \rightarrow \omega, s_{\sigma}: w_{\sigma \mid(|\sigma|-1)} \rightarrow \omega$ for $\sigma \neq \emptyset, s_{\sigma}(i)>\sigma(|\sigma|-1)$ for $i \in w_{\sigma \mid(|\sigma|-1)}$, and for all $x \in \omega^{\omega}, \omega=\operatorname{dom}\left(s_{\emptyset}\right) \cup$ $\bigcup_{n} w_{x \mid n}$. Clearly, $S$ is a subset of the Polish space $\left([\omega]^{<\omega} \times \omega^{[\omega]^{<\omega}}\right)^{\omega^{<\omega}}$ and a quick examination of its definition shows that it is $\boldsymbol{\Pi}_{1}^{1}$. Call a set $C \subseteq \omega^{\omega}$ nice if for some $\left(\left(w_{\sigma}, s_{\sigma}\right): \sigma \in \omega^{<\omega}\right) \in S$,

$$
C=\left\{y \in \omega^{\omega}: s_{\emptyset} \subseteq y \text { and } \exists x \in \omega^{\omega} \forall n \in \omega\left(y \mid w_{x \mid n}=s_{x \mid n+1}\right)\right\} .
$$

Now [BHS, Theorem 1.1] implies that a closed set is dominating precisely when it contains a nice set. Therefore,

$$
\begin{aligned}
H \in \mathrm{CND} \quad \text { iff } \quad & \forall\left(\left(w_{\sigma}, s_{\sigma}\right): \sigma \in \omega^{<\omega}\right) \in S \exists y \in \omega^{\omega} \\
& \left(y \notin H \text { and } s_{\emptyset} \subseteq y \text { and } \exists x \in \omega^{\omega} \forall n y \mid w_{x \mid n}=s_{x \mid n+1}\right) .
\end{aligned}
$$

Counting quantifiers, we see that this definition is $\boldsymbol{\Pi}_{2}^{1}$. 
Corollary 3.3. Let $G$ be a Polish group with an invariant metric. The family of all closed Haar null sets is Borel iff $G$ is locally compact.

Proof. The mapping $\Phi$ from Lemma 3.1 is a Borel mapping if $\mathcal{F}(G)$ and $\mathcal{F}\left(\omega^{\omega}\right)$ are equipped with the Effros Borel structure since this is the Borel structure generated by Fell's topology. So the corollary follows from Lemma 3.2. (The implication $\Leftarrow$ is standard.)

REMARK. The obvious estimation on the complexity of the set of closed Haar null subsets of a Polish group $G$ is that it is $\boldsymbol{\Sigma}_{2}^{1}$. Here is a calculation showing it. Let $P(G)$ be the set of all Borel probability measures on $G$. Then $P(G)$ with an appropriate topology forms a Polish space (see [K, 17.23]). The condition $\mu(g H h)=0$ is Borel in the Polish space of quadruples $(H, \mu, g, h) \in \mathcal{F}(G) \times P(G) \times G \times G$ (this follows from [K, 17.25]). Thus the condition

$$
H \in \mathcal{F}(G) \text { is Haar null iff } \exists \mu \in P(G) \forall g, h \in G \quad \mu(g H h)=0
$$

is $\boldsymbol{\Sigma}_{2}^{1}$. An argument as in Corollary 3.3, using Lemma 3.2 and the result of Hjorth quoted in the remark following Lemma 3.2, shows that the family of closed Haar null subsets of a Polish group, at least when this group admits an invariant metric, is $\boldsymbol{\Pi}_{1}^{1}$ - and $\boldsymbol{\Sigma}_{1}^{1}$-hard.

For two partially ordered sets $P, Q$ let $P \leq_{\mathrm{T}} Q(P$ is Tukey below $Q)$ if there is a mapping $f: P \rightarrow Q$, called a Tukey reduction, such that for any $q \in Q,\{p \in P: f(p) \leq q\}$ is bounded from above in $P$. In [F] Fremlin studied relations with respect to the Tukey order between partially ordered sets of the form $(I, \subseteq)$ where $I$ is an ideal and $\subseteq$ is the partial ordering of inclusion between sets in $I$. He also noticed that $P \leq_{\mathrm{T}} Q$ implies inequalities between certain cardinal coefficients associated with partially ordered sets, namely: $\operatorname{add}(Q) \leq \operatorname{add}(P)$ and $\operatorname{cf}(P) \leq \operatorname{cf}(Q)$ where $\operatorname{add}(P)$ is the smallest cardinality of an unbounded subset of $Q$ and $\operatorname{cf}(Q)$ is the smallest cardinality of a cofinal subset of $Q$, and similarly for $P$. Note that these cardinal coefficients are generalizations of additivity and cofinality of an ideal of sets. Fremlin showed in $[F]$ that Tukey inequality was behind the inequalities between appropriate cardinal coefficients of the ideal of Lebesgue measure zero sets and the ideal of meager sets on the real line which had been discovered earlier. Our function constructed in Theorem 2.1 gives a Tukey reduction between the ideals of Haar null sets and of non-dominating sets. We will need some notation. By $\mathcal{H N}(G)$ we denote the ideal of Haar null subsets of a Polish group $G$. When it is considered as a partial order it is understood that the order is inclusion. Similarly, if $\omega^{\omega}$ is viewed as a partial order, the order is $\leq^{*}$. Recall also that $\mathbf{b}$ is the smallest cardinality of an unbounded set in $\omega^{\omega}$ and $\mathbf{d}$ is the smallest cardinality of a dominating, that is, cofinal, subset of $\omega^{\omega}$. 
Corollary 3.4. Let $G$ be a Polish non-locally compact group with an invariant metric.

(i) $\omega^{\omega} \leq_{\mathrm{T}} \mathcal{H N}(G)$.

(ii) $\operatorname{add}(\mathcal{H N}(G)) \leq \mathbf{b}$ and $\mathbf{d} \leq \operatorname{cf}(\mathcal{H N}(G))$.

Proof. Let $f$ and $F$ be as in Theorem 2.1. Let $\phi: \omega^{\omega} \rightarrow \mathcal{H N}(G)$ be defined by

$$
\phi(x)=f^{-1}\left(\left\{y \in \omega^{\omega}: y(n) \leq x(n) \text { for infinitely many } n\right\}\right) .
$$

It follows easily from Theorem 2.1(ii) that $\phi$ is a Tukey reduction. Thus, (i) is established. We get (ii) above as a consequence of (i) by [F, Theorem 1J(a)] since $\mathbf{b}=\operatorname{add}\left(\omega^{\omega}\right)$ and $\mathbf{d}=\operatorname{cf}\left(\omega^{\omega}\right)$. (Point (ii) is easy to check directly from Theorem 2.1(ii) as well.)

The next corollary is to show that the known dissimilarities between Haar null sets on locally compact and non-locally compact groups can be easily deduced from Theorem 2.1. Point (i) below has been proved by Dougherty [D] and earlier by Christensen for abelian groups (implicit in [C1, Theorem 2]). Point (ii) is due to Solecki [S]. Earlier Dougherty [D] proved it for a large subclass of abelian groups.

Corollary 3.5. Let $G$ be a Polish non-locally compact group with an invariant metric.

(i) Each compact subset of $G$ is Haar null.

(ii) There exists a family of cardinality continuum of disjoint closed subsets of $G$ which are not Haar null.

Proof. Let $F$ and $f$ be as in Theorem 2.1.

(i) Let $K \subseteq G$ be compact. By Theorem 2.1, $g K \subseteq F$ for some $g \in G$. Then $f[g K]$ is a compact subset of $\omega^{\omega}$, so it is not dominating. Thus, by Theorem 2.1, $f^{-1}(f[g K]) \supseteq g K$ is Haar null.

(ii) Let $X_{n}, n \in \omega$, be disjoint infinite subsets of $\omega$ whose union is $\omega$. For $\alpha \in 2^{\omega}$, let

$$
A_{\alpha}=\left\{x \in \omega^{\omega}: x(k) \text { is even iff } k \in X_{n} \text { with } \alpha(n)=1\right\} .
$$

Then it is easy to see that the $A_{\alpha}$ 's are closed, dominating and pairwise disjoint. By Theorem 2.1, $f^{-1}\left(A_{\alpha}\right), \alpha \in 2^{\omega}$, are pairwise disjoint, closed, non-Haar null subsets of $G$.

Remarks. 1. In a recent paper Shi and Thompson [ST] show that for the group of all homeomorphisms of the unit interval, which is an important example of a Polish group not admitting an invariant metric, the conclusion of Corollary 3.5(ii) holds.

2. Corollary 3.5(ii) implies that the $\sigma$-complete Boolean algebra of Borel subsets of $G$ modulo the $\sigma$-ideal of Borel Haar null subsets of $G$ does not 
have the countable chain condition. Actually, the function $B \mapsto f^{-1}(B)$ induces an embedding, which preserves countable suprema, into this algebra of the $\sigma$-complete Boolean algebra of Borel subsets of $\omega^{\omega}$ modulo Borel nondominating sets.

4. Haar null sets in product groups. By Haar measures I mean left invariant Haar measures. The theorem in this section concerns Haar null sets in the product group $\prod_{n} G_{n}$ with each factor $G_{n}$ locally compact and points in the direction opposite to Theorem 2.1. It shows that when all but finitely many of the $G_{n}$ 's are amenable, then the $\sigma$-ideal of Haar null sets in $\prod_{n} G_{n}$ is closely connected to the Haar measures on the $G_{n}$ 's. In fact, Haar null sets in $\prod_{n} G_{n}$ are determined by measures defined on the factor groups and which are equivalent to Haar measures. (Recall that two Borel measures on a Polish space are equivalent if they have the same measure zero sets. Haar measures on a locally compact group are mutually equivalent.) This generalizes the theorem that Haar null sets on a locally compact group $G$ are precisely sets of Haar measure zero; simply take $G_{0}=G$ and $G_{n}=\{1\}$ for $n>0$.

Recall that a locally compact group $G$ is called amenable if it admits a left invariant mean on the space $L^{\infty}(G)$ of all essentially bounded complex functions measurable with respect to the Haar measure, that is, there exists a linear functional $m: L^{\infty}(G) \rightarrow \mathbb{R}$ such that essinf $f \leq m(f) \leq \operatorname{ess} \sup f$ for real $f \in L^{\infty}(G)$ and for any $g \in G$ and $f \in L^{\infty}(G), m(g f)=m(f)$ where ${ }_{g} f(h)=f\left(g^{-1} h\right)$. Let me mention here that this important class of groups contains all abelian and even exponentially bounded locally compact groups. To get a sense of the size of the class of amenable groups see $[\mathrm{P}$, Chapter 3].

Theorem 4.1. Let $G_{n}, n \in \omega$, be a sequence of locally compact Polish groups. If all but finitely many $G_{n}$ 's are amenable, then for each $n$ there is a sequence $\left(\mu_{k}^{n}\right)_{k}$ of probability Borel measures on $G_{n}$ such that

(i) each $\mu_{k}^{n}$ is equivalent to some (or, equivalently, all) Haar measures on $G_{n}$;

(ii) $A \subseteq \prod_{n} G_{n}$ is Haar null iff for some sequence $\left(k_{n}\right)$ and all $g, h \in$ $\prod_{n} G_{n}$,

$$
\left(\prod_{n} \mu_{k_{n}}^{n}\right)(g A h)=0
$$

Proof. We will need some notation. For $g \in \prod_{n} G_{n}$ and $n_{0} \in \omega$, let $g_{n_{0}}$, $g \mid n_{0}$, and $g \mid\left[n_{0}, \infty\right)$ be the projections of $g$ onto $G_{n_{0}}, \prod_{n<n_{0}} G_{n}, \prod_{n \geq n_{0}} G_{n}$, respectively. For a set $B \subseteq \prod_{n} G_{n}$ and $h \in \prod_{n<n_{0}} G_{n}$ let $B_{h}=\left\{g \mid\left[n_{0}, \infty\right)\right.$ : $g \in B$ and $\left.g \mid n_{0}=h\right\}$. 
Assume all $G_{n}$ with $n \geq p_{0}$ are amenable. Let $\lambda_{n}$ be a left invariant Haar measure on $G_{n}$. If $n<p_{0}$, for each $k$ let $\mu_{k}^{n}$ be some fixed Borel probability measure equivalent to $\lambda_{n}$. For $n \geq p_{0}, G_{n}$ is amenable, so by [P, Proposition 16.10], there exists a sequence $\left(A_{n, k}\right)_{k}$ of compact subsets of $G_{n}$ with positive Haar measure such that the following Følner condition is satisfied: given a compact set $L \subseteq G_{n}$ and $\varepsilon>0$, for all but finitely many $k \in \omega, \lambda_{n}\left(g A_{n, k} \triangle A_{n, k}\right) / \lambda_{n}\left(A_{n, k}\right)<\varepsilon$ for each $g \in L$. Here and below $B_{1} \triangle B_{2}$ stands for the symmetric difference $\left(B_{1} \backslash B_{2}\right) \cup\left(B_{2} \backslash B_{1}\right)$. Now let $\mu_{n}$ be a Borel probability measure on $G_{n}$ equivalent to $\lambda_{n}$. Let

$$
\lambda_{k}^{n}=\frac{1-1 / k}{\lambda_{n}\left(A_{n, k}\right)}\left(\lambda_{n} \mid A_{n, k}\right)
$$

and define

$$
\mu_{k}^{n}=\lambda_{k}^{n}+\frac{1}{k} \mu_{n}
$$

Obviously, each $\mu_{k}^{n}$ is a probability Borel measure equivalent to $\lambda_{n}$, so (i) is satisfied.

In (ii) only the direction from left to right needs proving. First we need the following claim.

Claim 1. Let $n \geq p_{0}$. For a given $L \subseteq G_{n}$ compact and $\varepsilon>0$, for all but finitely many $k$ 's, $\lambda_{k}^{n}\left(g A_{n, k}\right) \geq 1-\varepsilon$ for any $g \in L$.

Proof. Let $k$ be so large that $1 / k<\varepsilon / 2$ and $A_{n, k}$ satisfies the Følner condition for $L$ and $\varepsilon / 2$. Then

$$
\begin{aligned}
\lambda_{k}^{n}\left(g A_{n, k}\right) & =(1-1 / k) \frac{\lambda_{n}\left(g A_{n, k} h \cap A_{n, k}\right)}{\lambda_{n}\left(A_{n, k}\right)} \\
& \geq(1-\varepsilon / 2) \frac{\lambda_{n}\left(A_{n, k}\right)-\lambda_{n}\left(g A_{n, k} \triangle A_{n, k}\right)}{\lambda_{n}\left(A_{n, k}\right)} \\
& \geq(1-\varepsilon / 2)(1-\varepsilon / 2) \geq 1-\varepsilon .
\end{aligned}
$$

Let now $A \subseteq \prod_{n} G_{n}$ be universally measurable and Haar null. Let $\mu$ be a probability Borel measure such that $\mu(g A h)=0$ for all $g, h \in G$. Fix a compact set $K \subseteq \prod_{n} G_{n}$ with $\mu(K)>0$. Now using Claim 1, we can find a sequence $\left(k_{n}\right)$ so that $\sum_{n} 1 / k_{n}<\infty$ and for each $n \geq p_{0}$ and each $g_{n}$ in the projection of $K$ onto $G_{n}$, we have

$$
\lambda_{k_{n}}^{n}\left(g_{n} A_{n, k_{n}}\right) \geq 1-2^{-n} \quad \text { and } \quad \lambda_{k_{n}}^{n}\left(A_{n, k_{n}}\right) \geq 1-2^{-n} .
$$

Let $\nu=\prod_{n} \mu_{k_{n}}^{n}$. We claim that $\nu$ works. Assume towards contradiction that it does not; this implies that we can find $\bar{g}, \bar{h} \in \prod_{n} G_{n}$ such that

$$
\nu(\bar{g} A \bar{h}) \geq \varepsilon
$$


for some $\varepsilon>0$. Let $C=\bar{g} A \bar{h}$. Let

$$
\nu_{n}=\prod_{m<n} \mu_{k_{m}}^{m} \quad \text { and } \quad \nu^{n}=\prod_{m \geq n} \mu_{k_{m}}^{m}
$$

so $\nu=\nu_{n} \times \nu^{n}$. Pick $n_{0}$ so large that $n_{0} \geq \max \left(p_{0}, 1\right), \prod_{n \geq n_{0}}\left(1-2^{n}\right)$ $\geq 1-\varepsilon / 8$ and $\sum_{n \geq n_{0}} 1 / k_{n} \leq \varepsilon / 4$. By Fubini's theorem, from $(\overline{2})$ we have

$$
\nu_{n_{0}}\left(\left\{h \in \prod_{n<n_{0}} G_{n}: \nu^{n_{0}}\left(C_{h}\right) \geq \frac{3}{4} \varepsilon\right\}\right)>0 .
$$

Since $\nu_{n_{0}}$ is equivalent to the Haar measure on $\prod_{n<n_{0}} G_{n}$, translates of $\nu_{n_{0}}$-positive sets are $\nu_{n_{0}}$-positive. Hence, from the above inequality, we get

$$
\nu_{n_{0}}\left(h^{\prime}\left\{h \in \prod_{n<n_{0}} G_{n}: \nu^{n_{0}}\left(C_{h}\right) \geq \frac{3}{4} \varepsilon\right\}\right)>0 \quad \text { for any } h^{\prime} \in \prod_{n<n_{0}} G_{n}
$$

Claim 2. For every $g \in K^{-1}$ and every universally measurable $D \subseteq$ $\prod_{n \geq n_{0}} G_{n}$, if $\nu^{n_{0}}(D) \geq(3 / 4) \varepsilon$, then $\nu^{n_{0}}\left(\left(g \mid\left[n_{0}, \infty\right)\right) D\right) \geq \varepsilon / 4$.

Proof. Note first that by (1),

$$
\begin{aligned}
\left(\prod_{n \geq n_{0}} \lambda_{k_{n}}^{n}\right)\left(\left(g \mid\left[n_{0}, \infty\right)\right)^{-1} \prod_{n \geq n_{0}} A_{n, k_{n}}\right) & =\prod_{n \geq n_{0}} \lambda_{k_{n}}^{n}\left(g_{n}^{-1} A_{n, k_{n}}\right) \\
& \geq \prod_{n \geq n_{0}}\left(1-2^{-n}\right) \geq 1-\varepsilon / 8
\end{aligned}
$$

and similarly, again by (1),

$$
\left(\prod_{n \geq n_{0}} \lambda_{k_{n}}^{n}\right)\left(\prod_{n \geq n_{0}} A_{n, k_{n}}\right) \geq \prod_{m \geq n_{0}}\left(1-2^{-n}\right) \geq 1-\varepsilon / 8 .
$$

Using (4) and (5), we have

$$
\begin{aligned}
\nu^{n_{0}}(D) \leq & \left(\prod_{n \geq n_{0}} \lambda_{k_{n}}^{n}\right)(D)+\sum_{n \geq n_{0}} 1 / k_{n} \\
\leq & \left(\prod_{n \geq n_{0}} \lambda_{k_{n}}^{n}\right)\left(D \cap\left(g \mid\left[n_{0}, \infty\right)\right)^{-1} \prod_{n \geq n_{0}} A_{n, k_{n}} \cap \prod_{n \geq n_{0}} A_{n, k_{n}}\right) \\
& +\varepsilon / 8+\varepsilon / 8+\varepsilon / 4 .
\end{aligned}
$$

If we now let $B=D \cap\left(g \mid\left[n_{0}, \infty\right)\right)^{-1} \prod_{n \geq n_{0}} A_{n, k_{n}} \cap \prod_{n \geq n_{0}} A_{n, k_{n}}$, we see that both $B$ and $\left(g \mid\left[n_{0}, \infty\right)\right) B$ are included in $\prod_{n \geq n_{0}} A_{n, k_{n}}$, hence by the invariance of the Haar measure,

$$
\left(\prod_{n \geq n_{0}} \lambda_{k_{n}}^{n}\right)(B)=\left(\prod_{n \geq n_{0}} \lambda_{k_{n}}^{n}\right)\left(\left(g \mid\left[n_{0}, \infty\right)\right) B\right) .
$$


Thus continuing with (6), we get

$$
\begin{aligned}
\nu^{n_{0}}(D) \leq & \left(\prod_{n \geq n_{0}} \lambda_{k_{n}}^{n}\right)\left(\left(g \mid\left[n_{0}, \infty\right)\right) D\right. \\
& \left.\cap \prod_{n \geq n_{0}} A_{n, k_{n}} \cap\left(g \mid\left[n_{0}, \infty\right)\right) \prod_{n \geq n_{0}} A_{n, k_{n}}\right)+\varepsilon / 2 \\
\leq & \nu^{n_{0}}\left(\left(g \mid\left[n_{0}, \infty\right)\right) D\right)+\varepsilon / 2 .
\end{aligned}
$$

From this last estimate the claim follows immediately.

Now, using Claim 2, we estimate $\nu\left(g^{-1} C\right)$ for $g \in K$ (the last inequality, $>0$, follows from $(3))$ :

$$
\begin{aligned}
\nu\left(g^{-1} C\right) & =\int_{\prod_{n<n_{0}} G_{n}} \nu^{n_{0}}\left(\left(g^{-1} C\right)_{h}\right) d \nu_{n_{0}}(h) \\
& =\int_{\prod_{n<n_{0}} G_{n}} \nu^{n_{0}}\left(\left(g \mid\left[n_{0}, \infty\right)\right)^{-1}(C)_{\left(g \mid n_{0}\right) h}\right) d \nu_{n_{0}}(h) \\
& \geq \int_{\left\{h \in \prod_{n<n_{0}} G_{n}: \nu^{n_{0}}\left(C_{\left(g \mid n_{0}\right) h}\right) \geq 3 \varepsilon / 4\right\}} \frac{\varepsilon}{4} d \nu_{n_{0}}(h) \\
& =\frac{\varepsilon}{4} \nu_{n_{0}}\left(\left(g \mid n_{0}\right)^{-1}\left\{h \in \prod_{n<n_{0}} G_{n}: \nu^{n_{0}}\left(C_{h}\right) \geq 3 \varepsilon / 4\right\}\right)>0 .
\end{aligned}
$$

This means that

$$
\mu\left(\left\{g: \nu\left(g^{-1} \bar{g} A \bar{h}\right)>0\right\}\right)>0 .
$$

Now applying Fubini's theorem to the universally measurable set $\{(g, h) \in$ $\left.\prod_{n} G_{n} \times \prod_{n} G_{n}: g h \in \bar{g} A \bar{h}\right\}$ with the $g$-coordinate equipped with the measure $\mu$ and the $h$-coordinate with $\nu$, we get $\nu\left(\left\{h: \mu\left(\bar{g} A \bar{h} h^{-1}\right)>0\right\}\right)>0$, whence $\mu\left(\bar{g} A \bar{h} h^{-1}\right)>0$ for some $h$, contradiction.

\section{References}

[BHS] J. Brendle, G. Hjorth and O. Spinas, Regularity properties for dominating projective sets, Ann. Pure Appl. Logic 72 (1995), 291-307.

[C1] J. P. R. Christensen, On sets of Haar measure zero in abelian Polish groups, Israel J. Math. 13 (1972), 255-260.

[C2] - Measure theoretic zero sets in infinite dimensional spaces and applications to differentiability of Lipschitz mappings, Publ. Dép. Math. (Lyon) 10 (1973), 29-39.

[D] R. Dougherty, Examples of nonshy sets, Fund. Math. 144 (1994), 73-88.

[DM] R. Dougherty and J. Mycielski, The prevalence of permutations with infinite cycles, ibid. 144 (1994), 89-94.

[E] R. Engelking, General Topology, Heldermann, 1989. 
[F] D. H. Fremlin, The partially ordered sets of measure theory and Tukey's ordering, Note Mat. 11 (1991), 177-214.

[H] G. Hjorth, a handwritten note.

$[\mathrm{Hu}] \quad$ B. R. Hunt, The prevalence of continuous nowhere differentiable functions, Proc. Amer. Math. Soc. 122 (1994), 711-717.

[HSY] B. R. Hunt, T. Sauer and J. A. Yorke, Prevalence: a translation invariant "almost every" on infinite-dimensional spaces, Bull. Amer. Math. Soc. (N.S.) 27 (1992), $217-238$.

[K] A. S. Kechris, Classical Descriptive Set Theory, Springer, 1995.

[KLW] A. S. Kechris, A. Louveau and W. H. Woodin, The structure of $\sigma$-ideals of compact sets, Trans. Amer. Math. Soc. 301 (1987), 263-288.

[LZ] C. Laflamme and J.-P. Zhu, The Rudin-Blass ordering on ultrafilters, J. Symbolic Logic 63 (1998), 584-592.

[Ma] P. Mankiewicz, On the differentiability of Lipschitz mappings in Fréchet spaces, Studia Math. 45 (1973), 15-29.

[M1] E. Matouškova, Convexity and Haar null sets, Proc. Amer. Math. Soc. 125 (1997), 1793-1799.

[M2] - The Banach-Saks property and Haar null sets, Comment. Math. Univ. Carolin. 39 (1998), 71-80.

[M3] - , Translating finite sets into convex sets, preprint, 1999.

[MS] E. Matouškova and C. Stegall, A characterization of reflexive Banach spaces, Proc. Amer. Math. Soc. 124 (1996), 1083-1090.

[P] J.-P. Pier, Amenable Locally Compact Groups, Wiley, 1984.

[ST] H. Shi and B. S. Thompson, Haar null sets in the space of automorphisms on [0,1], Real Anal. Exchange 24 (1998/99), 337-350.

[S] S. Solecki, Haar null sets, Fund. Math. 149 (1996), 205-210.

Department of Mathematics

Indiana University

Bloomington, IN 47405, U.S.A.

E-mail: ssolecki@indiana.edu 